\title{
An Improved Beamforming Schemes in Coordinated Multi-Point Systems
}

\author{
Hongtao Guo \\ North China University of Water Resources and Electric Power, \\ Zhengzhou 450045, Henan, China. \\ htguo2000@163.com
}

\begin{abstract}
The capacity of modern cellular wireless networks is mainly limited by inter-cell interference (ICI). Coordinated multi-point (CoMP) transmission and reception were adopted as key techniques for Long Term Evolution-Advanced (LTE-A) as they are capable of mitigating ICI through cooperation between several cell sites. Therefore, CoMP techniques provide a significant improvement in cell-edge and average throughput. For downlink CoMP, most research work mainly focuses on joint processing/transmission (CoMP-JP) mode. However this mode needs the access points (AP) to share the UEs' data and channel state information (CSI) simultaneously, resulting in a high overhead on the network. Coordinated beamforming (CoMP-CBF), which can avoid the data information exchange is a better solution to reduce the overhead.

In this paper, an improved cooperative beamforming schemes for downlink Coordinated Multi-point System is described. The proposed improved beamforming schemes rely on the principal eigen-vector feedback schemes (e.g. codebook based). Although the description of the beamforming schemes is not a part of specification, the detailed information on the used coordinated beamforming approaches is important for performance evaluation and calibration of the results during CoMP phase. System-level simulation results demonstrate that the proposed scheme can significantly reduce the ICI, and it performs well even with imperfect channel-state information (CSI) at the transmitter.
\end{abstract}

Keywords: CoMP, LTE-advanced, Coordinated Beamforming, eigen-vector

\section{Introduction}

Long Term Evolution Advanced (LTE-A) system has been proposed by the 3rd Generation Partnership Project (3GPP). Multiple-input multiple-output (MIMO) technique can effectively increase system peak spectrum efficiency in wireless communication and has been applied in LTE [1]. However, the cell-edge spectrum efficiency can't be promoted significantly because of the severe inter-cell interference (ICI). Which will cause severe degradation of throughput and could not be decreased by heightening the transmit power of desired signals. Consequently, ICI becomes dominating channel impairment and degrades the overall system performance significantly. In order to obtain better system performance, a promising technique, Coordinated Multi-point Transmission (CoMP) is added in the RANI \#53bis meeting [2].

There are two classes of downlink CoMP strategy: joint processing/transmission (CoMP-JP) and coordinated scheduling/beamforming (CoMP-CBF) [3]. In CoMP-JP mode, data and channel state information (CSI) from multi-cell are shared in the CoMP set for joint transmission to user equipment (UE). While in CoMP-CBF mode, the data information to UEs are just transmitted by their serving cell but other cells in the CoMP set only coordinate their transmission to reduce the ICI. 
Recently, the problem of intra-cell interference mitigation has been thoroughly discussed for MU-MIMO schemes. In Releases 8-10 the codebook based feedback has been adopted to support MU-MIMO beamforming techniques. Since the requirements on the interference suppression level for intra-cell (MU-MIMO) beamforming schemes are usually more stringent than for inter-cell beamforming schemes (CoMP), the codebook based feedback should be considered with a higher priority to support CoMP. Therefore in this paper, we focus on the description of the coordinated beamforming schemes operating on the principle eigen-vector or codebook based feedbacks.

\section{System Model}

For simplicity, two coordinating cells denoted as eNB1 and eNB2 are considered as shown in Figure 1. eNB1 and eNB2 are connected with each other via a backhaul link and employ multiple transmit antennas with adaptive beamforming and coordination. In deployments with low reuse factor (e.g., reuse-1) eNB1 (serving UE1) and eNB2 (serving UE2) may create significant interference at each other's UEs due to simultaneous downlink transmission in the same time-frequency resources [4].

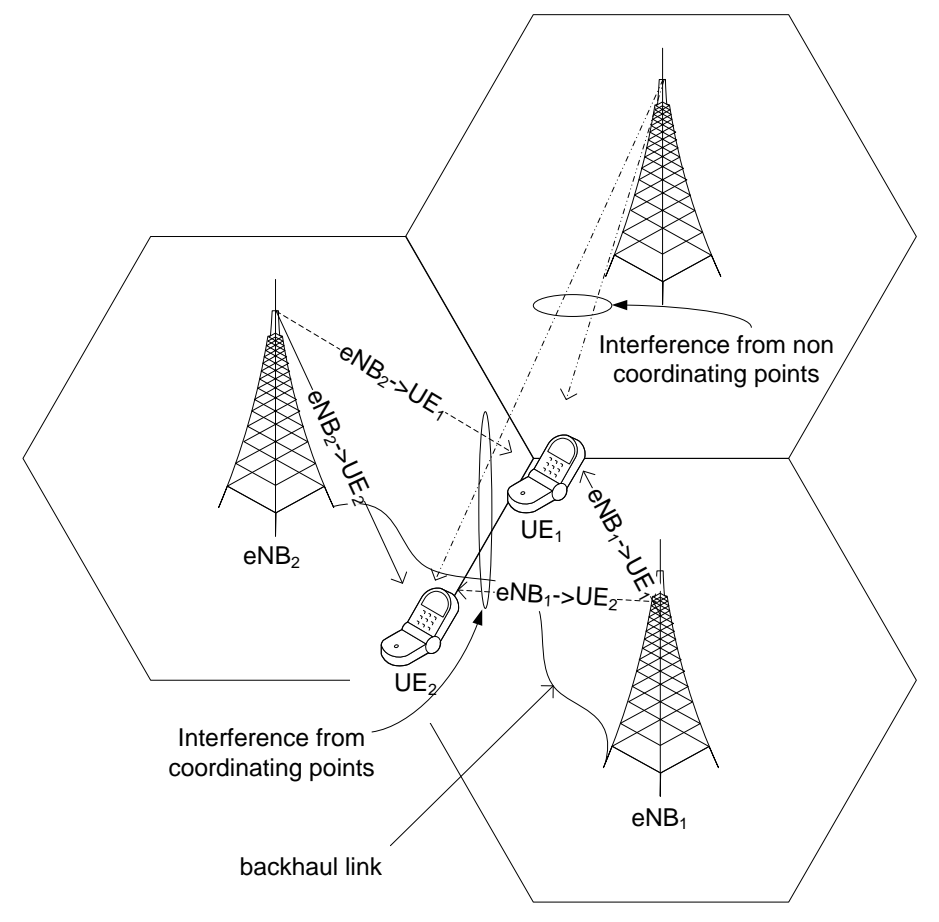

Figure 1. Cellular System Employing CoMP Beamforming

The idea of beamforming coordination relies on the possible adjustment of the beamforming vectors at the eNB1 and eNB2 to minimize the negative impact from mutual interference. The principles of beamforming adjustment to cope with interference has been developed and discussed in the context of single-cell multi-user MIMO (MUMIMO) beamforming schemes, where the performance due to simultaneous transmission is usually limited by the level of inter-user (intra-cell) interference [5]. In MU-MIMO systems, the usage of beamforming schemes with effective interference suppression to other users is very critical for the overall system performance. In a coordinated multipoint system, inter-user interference also exists; however, in contrast to the conventional single-cell MU-MIMO systems, interference suppression should be conducted for the users of the other cells (inter-cell interference). In this case, the principles of the conventional (single-point) multi-user MIMO beamforming schemes (e.g. Zero-forcing, 
Regularized Zero-forcing, etc.) can be utilized to some extent for multi-point coordinated beamforming [6].

\section{Conventional and Regularized Zero-Forcing Beamforming Schemes}

For the sake of simplicity let's consider interference adjustment at the eNB1. Let $\mathbf{v}^{\left(\mathrm{eNB}_{1} \rightarrow \mathrm{UE}_{1}\right)}$ and $\mathbf{v}^{\left(\mathrm{eNB}_{1} \rightarrow \mathrm{UE}_{2}\right)}$ denote the principal eigenvectors fed back from UE1 and UE2, respectively, with respect to their corresponding channels to eNB1. $\mathbf{v}^{\left(\mathrm{eNB}_{1} \rightarrow \mathrm{UE}_{2}\right)}$ approximating the interference space of eNB1 can be reported from UE2 to its serving eNB2 and provided to eNB1 by using the backhaul link between eNB1 and eNB2. Based on the feedback from UE1 and UE2 the eNB1 adjusts its beamforming vector in order to minimize interference to UE2 served by eNB2. The beamforming adjustment at the eNB1 can be performed for example by using the multi-user Zero-Forcing (MUZF) procedure as follows:

$\mathbf{W}=\left[\mathbf{w}_{1}^{\left(\mathrm{eNB}_{1} \rightarrow \mathrm{UE}_{1}\right)} \mathbf{w}_{2}\right]=\mathbf{V}\left(\mathbf{V}^{H} \mathbf{V}\right)^{-1}$

Matrix $\mathbf{V}=\left[\begin{array}{ll}\mathbf{v}^{\left(\mathrm{eNB}_{1} \rightarrow \mathrm{UE}_{1}\right)} & \mathbf{v}^{\left(\mathrm{eNB}_{1} \rightarrow \mathrm{UE}_{2}\right)}\end{array}\right]$ is the concatenation of the UE1 and UE2 principal eigenvectors with respect to eNB1. The multi-point beamforming vector of the serving eNB1 in this case corresponds to the first column of the matrix $\mathbf{W}$ in (1), denoted as $\mathbf{w}_{1}^{\left(\mathrm{eNB}_{1} \rightarrow \mathrm{UE}_{1}\right)}$. The vector corresponding to UE2 served by the neighboring eNB2, denoted as $\mathbf{w}_{2}$ in (1), is not utilized by eNB1 for data transmission. It can be seen from the above procedure that the feedback from UE2 (served by eNB2) regarding the interference from eNB1 is accounted by eNB1 during the multi-user beamforming calculation in the same way as the feedback from all other UEs served by eNB1 [7]. However, in contrast to the conventional single-point MU-MIMO scheme, no data transmission from eNB1 to the UE2 is performed after the beamforming calculation. It can be also seen from (1) that due to zero-forcing operation the calculated beamforming vector $\mathbf{w}_{1}^{\left(\mathrm{eNB}_{1} \rightarrow \mathrm{UE}_{1}\right)}$ of eNB1 is orthogonal to the principal eigenvector $\mathbf{v}^{\left(\mathrm{eNB}_{1} \rightarrow \mathrm{UE}_{2}\right)}$ of the interfering channel, thus minimizing the interference impact from eNB1 to the neighboring cell. Figure 2 illustrates the beamforming adjustment procedure at the eNB1.

In Figure 2, the two UEs are located at angles 550 and -700 , respectively. The antenna pattern corresponding to the eNB1 $\rightarrow$ UE2 vector (which approximates the interference space) is shown in red color. The antenna pattern for eNB1 $\rightarrow$ UE1 beamforming without coordination is shown in blue color. It can be seen that without beamforming coordination significant interference is created at the direction of -700 where UE2 is located. The antenna pattern for the vector after beamforming adjustment procedure is shown in green color. It can be observed that after zero-forcing the beamforming vector for the link eNB1 $\rightarrow$ UE1 is selected in such a way that a null is placed towards the direction to UE2 served in the neighboring eNB2, so that the interference impact from eNB1 to UE2 is effectively suppressed. 


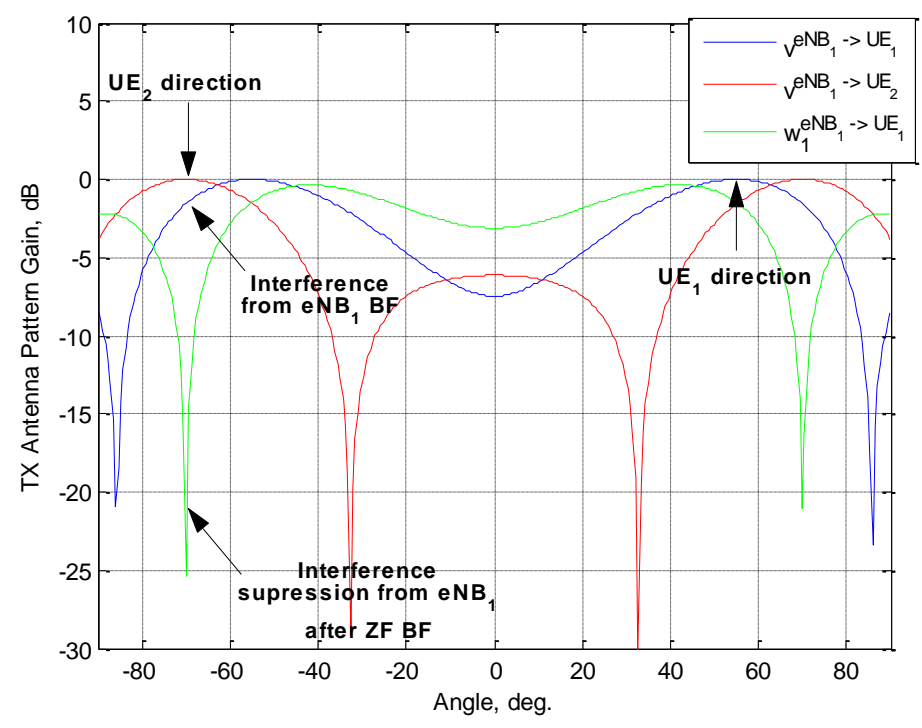

\section{Figure 2. Illustration of the Beamforming Adjustment Procedure for Cooperative Beamforming}

Interference suppression to other cells is generally performed at the cost of antenna pattern gain reduction in the direction of the served UE1, see Figure 2. Depending on the ratio of the interference power from eNB1 to the interference power from other cells, perfect interference suppression may not be required. Relaxing the perfect interference suppression criterion in the zero-forcing procedure can minimize the negative impact from antenna pattern gain reduction. To achieve such a goal in single-cell MU-MIMO beamforming, a regularization term is usually applied, which balances the interference power generated to the other user with the interference power from other cells. Such a regularization principle can be also utilized in the zero-forcing procedure for coordinated beamforming by allowing some level of interference from the cooperating cell [8]. In a similar way, the level of the generated interference from the cooperating cell will not substantially exceed the level of interference power from other cells. The equation for the calculation of the regularized zero-forcing beamforming vector can be represented in the follow:

$$
\mathbf{W}=\left[\begin{array}{ll}
\mathbf{w}_{1}^{\left(\mathrm{eNB}_{1} \rightarrow \mathrm{UE}_{1}\right)} & \mathbf{w}_{2}
\end{array}\right]=\mathbf{V}\left(\mathbf{V}^{H} \mathbf{V}+\left(\begin{array}{cc}
\gamma_{1}^{-1} & 0 \\
0 & \gamma_{2}^{-1}
\end{array}\right)\right)^{-1}
$$

where $\gamma_{i}$ can be selected, for example, as the average SINR on the eNB1 to UEi link with the interference power accounting only for non-cooperative (outer) cells. It should be noted that the required vector $\mathbf{w}_{1}^{\left(\mathrm{eNB}_{1} \rightarrow \mathrm{UE}_{1}\right)}$ only depends on parameter $\gamma_{2}$ which defines the severity of eNB1 interference to the neighboring cell.

An example of the antenna pattern gain reduction and interference suppression level for regularized $\mathrm{ZF}$ beamforming is shown in Figure 3 for different combinations of parameters $\gamma_{1}$ and $\gamma_{2}$. It can be seen that for the weak interference scenario (small to moderate values of parameter $\gamma_{2}$ ), regularized ZF beamforming does not substantially modify the reported vector, so that the scalar product of the calculated $\mathbf{w}_{1}^{\left(\mathrm{eNB}_{1} \rightarrow \mathrm{UE}_{1}\right)}$ with the reported $\mathbf{v}^{\left(\mathrm{eNB}_{1} \rightarrow \mathrm{UE}_{1}\right)}$ remains close to one. On the other hand, when the generated 
interference to the neighboring cell is high, the calculated vector $\mathbf{w}_{1}^{\left(\mathrm{eNB}_{1} \rightarrow \mathrm{UE}_{1}\right)}$ converges to the zero-forcing beamforming vector which perfectly cancels the interference to the neighboring cell. In this case, the vector $\mathbf{w}_{1}^{\left(\mathrm{eNB}_{1} \rightarrow \mathrm{UE}_{1}\right)}$ is subject to more significant transformations. It can be also seen from the Figure 3 that the calculated beamforming vector $\mathbf{w}_{1}^{\left(\mathrm{eNB}_{1} \rightarrow \mathrm{UE}_{1}\right)}$ is independent from parameter $\gamma_{1}$ which may reduce the amount of feedback information required for the calculation of the beamforming vectors [9-10].

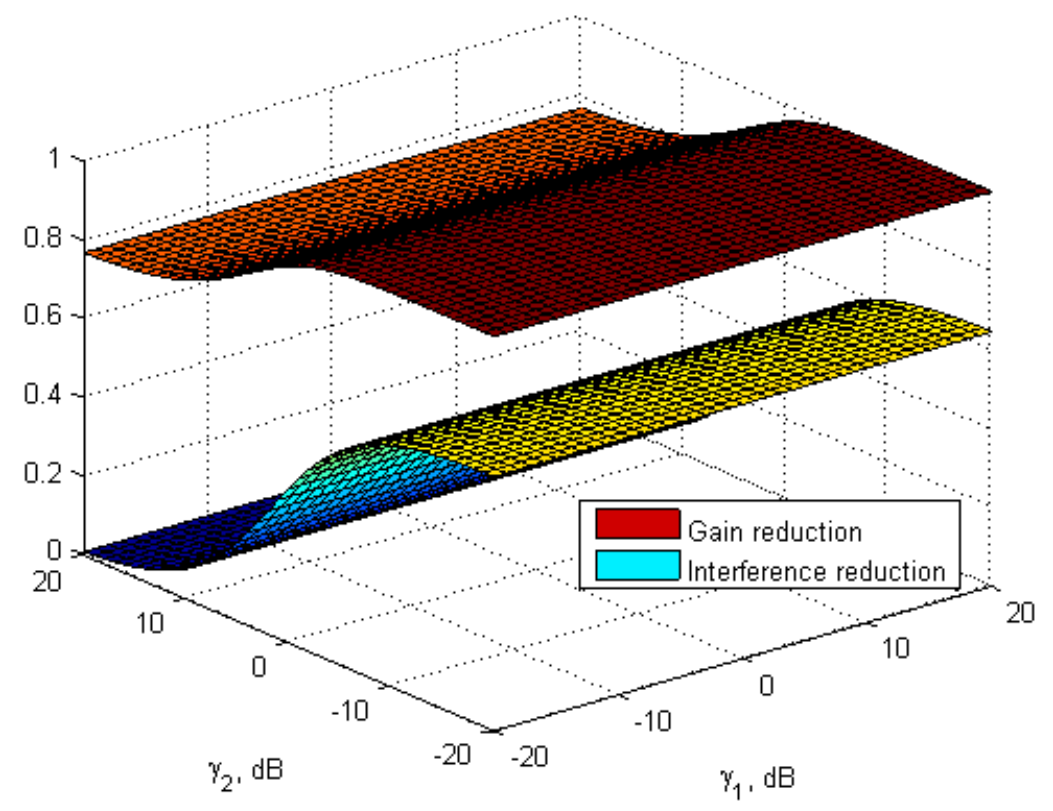

Figure 3. Antenna Pattern Gain Reduction and Interference Cancellation vs. $\gamma_{1}$ and $\gamma_{2}$

\section{An Improved Beamforming Schemes}

The problem of the conventional and regularized zero-forcing beamforming schemes lies in the capability of supporting optimal multi-rank beamforming to the UE. Generalization of zero-forcing beamforming supporting multiple-rank transmission to the UE can be obtained by using beamforming schemes based on the QR decomposition [11].

Considering the same model of the two cooperating eNBs, see Figure 1, the multi-rank beamforming matrix can be obtained from the QR decomposition of the concatenated matrix $\mathbf{V}$. The matrix is obtained by the concatenation of the multi-rank principal eigenvectors reported by UE2 and UE1 to the eNB1. The QR-decomposition of matrix $\mathbf{V}$ can be represented as follow:

$$
\mathbf{V}=\left[\begin{array}{ll}
\mathbf{V}^{\left(\mathrm{eNB}_{1} \rightarrow \mathrm{UE}_{2}\right)} & \mathbf{V}^{\left(\mathrm{eNB}_{1} \rightarrow \mathrm{UE}_{1}\right)}
\end{array}\right]=\mathbf{Q R}
$$

$\mathbf{Q}$ is a unitary beamforming matrix. $\mathbf{R}$ is an upper triangular matrix. The desired unitary beamforming matrix of eNB1 supporting multi-rank beamforming resides in the calculated matrix $\mathbf{Q}=\left[\begin{array}{ll}\mathbf{Q}^{\left(\mathrm{eNB}_{1} \rightarrow \mathrm{UE}_{2}\right)} & \mathbf{Q}^{\left(\mathrm{eNB}_{1} \rightarrow \mathrm{UE}_{1}\right)}\end{array}\right]$ on the same columns as in matrix $\mathrm{V}$. After 
multiplying (3) by $\mathbf{Q}^{H}$ from the left side, it can be verified that the beamforming matrix $\mathbf{Q}^{\left(\mathrm{eNB}_{1} \rightarrow \mathrm{UE}_{1}\right)}$ is orthogonal to the principal eigenvector $\mathbf{V}^{\left(\mathrm{eNB}_{1} \rightarrow \mathrm{UE}_{2}\right)}$, i.e.,

$$
\mathbf{Q}^{\left(\mathrm{eNB}_{1} \rightarrow \mathrm{UE}_{1}\right) H} \mathbf{V}^{\left(\mathrm{eNB}_{1} \rightarrow \mathrm{UE}_{2}\right)}=\mathbf{0}
$$

Sub-matrix $\mathbf{Q}^{\left(\mathrm{eNB}_{1} \rightarrow \mathrm{UE}_{1}\right)}$ satisfies the unitary condition (required for optimal multi-rank beamforming per each UE) due to the unitary structure of the calculated beamforming matrix $\mathbf{Q}$, i.e.,

$$
\mathbf{Q}^{\left(\mathrm{eNB}_{1} \rightarrow \mathrm{UE}_{1}\right) H} \mathbf{Q}^{\left(\mathrm{eNB}_{1} \rightarrow \mathrm{UE}_{1}\right)}=\mathbf{I}
$$

Figure 4 illustrates the QR beamforming adjustment procedure at the eNB1 for rank-2 transmission to UE1 and rank-1 approximation of the interference space to UE2.

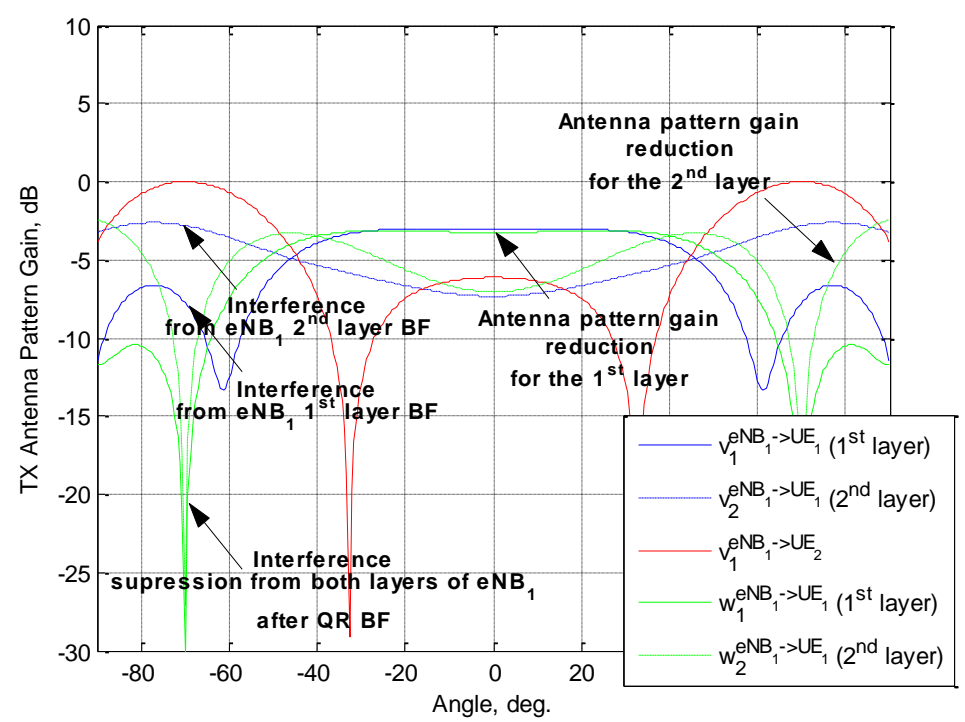

Figure 4. Illustration of QR Beamforming for Cooperative Beamforming

In Figure 4 the antenna pattern for eNB1 $\rightarrow$ UE1 without coordination is shown with the blue solid line for the 1st layer and the blue dashed line for the 2 nd layer. The antenna pattern corresponding to eNB1 $\rightarrow \mathrm{UE} 2$ approximating the interference space is shown in red color. It can be seen from the figure that without beamforming coordination significant interference is created to UE2 by both spatial layers of UE1 [12]. However, after QR beamforming the beamforming vectors for the 1st and 2nd spatial layers (solid and dashed green lines, respectively) are modified in such a way that nulls are placed towards the direction to UE2 served by the neighboring eNB2, which effectively suppresses the interference impact from eNB1 to UE2.

As it was pointed out earlier in this section, interference suppression to other cells is performed at the cost of antenna pattern gain reduction in the direction of the served UE1. From Figure 4 it can be seen that although the antenna pattern gain reduction for the 1st layer is not significant, the impact to the 2 nd layer is more remarkable. Such a difference can be explained by the placement (orthogonalization) order of the beamforming vectors of each layer. Since after the QR decomposition each column of the matrix Q must be orthogonal to all preceding columns of matrix $\mathrm{V}$, the last columns of matrix $\mathrm{Q}$ are subject to more significant beamforming vector transformations (a higher number of orthogonality conditions have to be satisfied than in preceding vectors). 
The difference in the antenna pattern gain reductions for each layer dictates the preferred placement order in matrix $\mathrm{V}$ for the principal eigenvectors representing the serving channel. Since the stronger layer is usually conveying the higher amount of information, substantial modifications to the corresponding beamforming vector are not desirable. In this case, it is preferable to order the columns of matrix $\mathbf{V}^{\left(\mathrm{eNB}_{1} \rightarrow \mathrm{UE}_{1}\right)}$ according to their effective SINRs. In the two-layer system, the per layer SINR can be determined at the eNB from the codeword-specific CQI reports thanks to multi-codeword coding and feedback employed in LTE systems.

Similar to regularized zero-forcing, a regularization extension can be also introduced to the QR beamforming scheme as follows

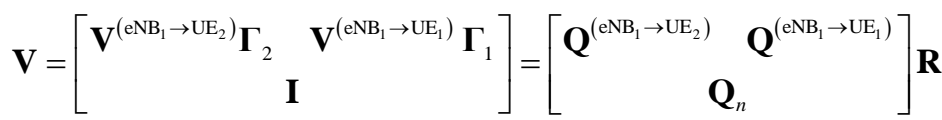

where I is an identity matrix with the dimensions equal to the number of columns of matrix $\mathrm{V}$, matrix $\Gamma_{i}$ is a diagonal matrix of dimensions $N_{i} \times N_{i}$ with $N_{i}$ being the rank of matrix $\mathbf{V}^{\left(\mathrm{eNB}_{1} \rightarrow \mathrm{UE}_{i}\right)}$ :

Frequency offset estimation value:

$\boldsymbol{\Gamma}_{i}=\left[\begin{array}{ccc}\gamma_{i}^{(1)} & 0 & 0 \\ 0 & \ddots & 0 \\ 0 & 0 & \gamma_{i}^{\left(N_{i}\right)}\end{array}\right]$

The diagonal elements $\gamma_{i}^{(j)}$ of matrix $\Gamma_{i}$ contain the SINR values for each layer $\mathrm{j}$ corresponding to the link from eNB1 to UEi. The interference power in the per-layer SINR accounts only for non-cooperative cells. Similar to the regularized ZF scheme, only matrix $\Gamma_{2}$ (corresponding to the SINR of the neighboring cell) is required.

It should be pointed out that the vectors of sub-matrix $\mathbf{Q}^{\left(\mathrm{eNB}_{1} \rightarrow \mathrm{UE}_{1}\right)}$ are not normalized and the norm of the beamforming matrix is an indicator of whether the channels of the selected user group are "compatible" with each other. Therefore, in order to avoid inefficient user combinations it is recommended to use matrix $\mathbf{Q}^{\left(\mathrm{eNB}_{1} \rightarrow \mathrm{UE}_{1}\right)}$ without normalization for the scheduling operation [13]. However, to avoid partial power loading and fluctuating interference power in the network, the calculated coordinated beamforming vectors of matrix $\mathbf{Q}^{\left(\mathrm{eNB}_{1} \rightarrow \mathrm{UE}_{1}\right)}$ for the selected user group should be normalized when it is used for the actual data transmission.

The coordinated beamforming schemes are presented assuming SU-MIMO transmission from serving eNB1. However, the beamforming equations can be easily extended to support MU-MIMO transmission as well. Multiple users can be accounted for in the beamforming scheme by expanding the concatenation procedure to the principal eigenvector reports from other users of the same cell.

\section{Conclusion}

In this paper, we have proposed an improved coordinated beamforming schemes based on the eigen-vector feedback for downlink CoMP systems. The presented schemes reuse the beamforming principles of single-cell MU-MIMO beamforming schemes and can rely on the codebook based feedback framework already supported in Releases 8-10. The 
proposed scheme can significantly reduce the ICI overhead on the network, and it performs well even with imperfect CSI at the transmitter.

\section{Acknowledgements}

This work was supported by the Key Science and Technology Program of He'nan Province of China under grant No 132102210044. It was also supported by Foundation of He'nan Educational Committee under grant No 14A520010.

\section{References}

[1] M. B. Naik, S. D. M. Mehata and B. D. Bhavani, "GCD Matrix based PAPR Reduction Technique for OFDM System", [J]. International Journal of Signal Processing, Image Processing and Pattern Recognition, vol. 7, no. 4, (2014), pp. 177-186.

[2] Z. Huang, B. Li and M. Liu, "Coordinated Beamforming of CoMP with limited feedback", [C]//Network Computing and Information Security (NCIS), 2011 International Conference on. IEEE, vol. 1, (2011), pp. 138-141.

[3] Z.Huang, B. Li and MLiu, "Coordinated Beamforming of CoMP with Limited Feedback", 2011 Internati onal Conference on Network Computing and Information Security, (2011).

[4] L. Qiang, Y. Yang and F. Shu, "Coordinated beamforming in downlink CoMP transmission system", $[\mathrm{C}] / /$ Communications and Networking in China (CHINACOM), 2010 5th International ICST Conference on. IEEE, (2010), pp. 1-5.

[5] Y. Huang, G. Zheng and M. Bengtsson, "Distributed multicell beamforming with limited intercell coordination",[J]. Signal Processing, IEEE Transactions on, vol. 59, no. 2, (2011), pp. 728-738.

[6] W. Yu, T. Kwon and C. Shin, "Multicell coordination via joint scheduling, beamforming, and power spectrum adaptation", [J]. Wireless Communications, IEEE Transactions on, (2013), vol. 12, no. 7, pp. $1-14$

[7] H. Je, H. Lee and K. Kwak, "Long-term channel information-based CoMP beamforming in LTEAdvanced systems", [C]//Global Telecommunications Conference (GLOBECOM 2011). IEEE, (2011), pp.1-6.

[8] Y. Huang and G. Zheng and M. Bengtsson, "Distributed multicell beamforming design approaching pareto boundary with max-min fairness", [J]. Wireless Communications, IEEE Transactions on, vol. 11, no. 8, (2012), pp. 2921-2933.

[9] J. Parikh and A. Basu, "LTE Advanced: The 4G mobile broadband technology", [J]. spectrum, (2011), vol. 5 , no. 2.5 , pp. 30 .

[10] T. Zhou, M. Peng and W. Wang, "Low-complexity coordinated beamforming for downlink multicell SDMA/OFDM systems", [J]. Vehicular Technology, IEEE Transactions on, (2013), vol. 62, no. 1, pp. 247-255.

[11] H. Kim, H. Yu and Y. Sung, "An efficient algorithm for zero-forcing coordinated beamforming"[J]. Communications Letters, IEEE, (2012), vol.16, no. 7, pp. 994-997.

[12] U. Jang, K. Y. Lee and K. S. Cho, "Transmit beamforming based inter-cell interference alignment and user selection with CoMP”, [C]//Vehicular Technology Conference Fall (VTC 2010-Fall), 2010 IEEE 72nd. IEEE, (2010), pp. 1-5.

[13] Z Huang, B. Li and M. Liu Coordinated Beamforming of CoMP with limited feedback[C]//Network Computing and Information Security (NCIS), 2011 International Conference on. IEEE, (2011), vol. 1, pp. 138-141.

\section{Author}

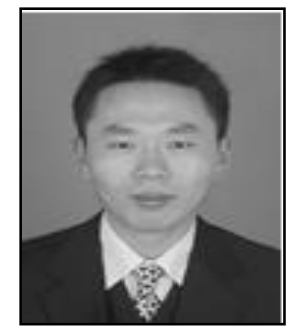

GUO Hong-tao, he received his MS degree in computer science from HUST (Hua-zhong University of Science and Technology), Wuhan, China in 2005. He is currently a lecturer in the software institute at North China University of Water Resources and Electric Power. He has more than 10 technical publications as well as about 3 patents. His current research interests include wireless communication system. 